This item was submitted to Loughborough's Research Repository by the author.

Items in Figshare are protected by copyright, with all rights reserved, unless otherwise indicated.

\title{
Coordinated trajectory planning for efficient communication relay using multiple UAVs
}

\section{PLEASE CITE THE PUBLISHED VERSION}

http://dx.doi.org/10.1016/j.conengprac.2014.04.003

\section{PUBLISHER}

(c) Elsevier Ltd

\section{VERSION}

AM (Accepted Manuscript)

\section{PUBLISHER STATEMENT}

This work is made available according to the conditions of the Creative Commons Attribution-NonCommercialNoDerivatives 4.0 International (CC BY-NC-ND 4.0) licence. Full details of this licence are available at: https://creativecommons.org/licenses/by-nc-nd/4.0/

\section{LICENCE}

CC BY-NC-ND 4.0

\section{REPOSITORY RECORD}

Kim, Seungkeun, Hyondong Oh, Jinyoung Suk, and Antonios Tsourdos. 2015. "Coordinated Trajectory Planning for Efficient Communication Relay Using Multiple Uavs”. figshare. https://hdl.handle.net/2134/17842. 


\title{
Coordinated Trajectory Planning for Efficient Communication Relay Using Multiple UAVs
}

\author{
Seungkeun $\mathrm{Kim}^{\mathrm{a}}$, Hyondong $\mathrm{Oh}^{\mathrm{b}, *}$, Jinyoung $\mathrm{Suk}^{\mathrm{a}}$, Antonios Tsourdos ${ }^{\mathrm{c}}$ \\ ${ }^{a}$ Department of Aerospace Engineering, Chungnam National University, 99 Daehak-ro, \\ Yuseong-gu, Daejeon 305-764, Korea \\ ${ }^{b}$ Department of Computing, University of Surrey, Guildford, Surrey, GU2 7XH, UK \\ ${ }^{c}$ Division of Engineering Sciences, Cranfield University, Cranfield, MK43 OAL, UK
}

\begin{abstract}
This paper investigates the use of small UAVs as communication relay nodes for expanding communications links and improving communication quality for a fleet of naval vessels. This paper firstly deals with the UAV deployment for stationary communication nodes, and then, proposes a decentralised nonlinear model predictive trajectory planning strategy for a dynamic environment. By exploiting motion estimates of vessels and states of UAVs, the trajectory planning algorithm finds a control input sequence optimising network connectivity over a certain time horizon. Numerical simulations are performed for both stationary and manoeuvring vessels to verify the feasibility and benefit of the proposed approach.

Keywords: Unmanned Aerial Vehicles, Communications Relay, Trajectory Planning, Global Message Connectivity, Minimum Spanning Tree.
\end{abstract}

\section{Introduction}

Traditionally communication relay was considered a secondary mission on a platform deployed on another main mission. However, with the advent of lightweight,

\footnotetext{
${ }^{*}$ Corresponding author

Email address: h. ohesurrey.ac.uk (Hyondong Oh)
} 
robust and autonomous platforms as well as wireless networking technologies, UAVs (unmanned aerial vehicles) can now perform this relay mission. UAV communication relay is intended to replace the legacy radios currently being used on tactical size UAVs such as Predator, Fire Scout, and Hunter, among others [1]. Regarding the airborne communication relay payload, Northrop Grumman developed a communication relay package to provide four communication software programmable channels, which can be configured to provide ground to ground, air to air, or ground to air relay [2]. Thales is also developing a dedicated version of the LMAR (Lightweight Multiband Airborne Radio) for the British Watchkeeper program [3]. This study is motivated by using such a capability for expanding communication links and improving communication quality, primarily for a fleet of ground or navy vessels. An airborne relay can effectively connect to units operating over the horizon, beyond normal communication range, or under limited satellite communication environments. However, even if the equipment development is relatively mature, and considerable research on a mobile ad-hoc networking has been performed for ground robot teams $[4,5,6]$, where to locate UAVs for efficient relay is still a pending problem due to UAV's dynamic and operational constraints.

The feasibility study to use UAVs as communication relay is performed mainly in support of a battlefield information transmission system initiative [7]. The main objective of this program was to provide beyond line-of-sight communications within an area of operations without using scarce satellite resources. Cerasoli [8] assessed the practical effectiveness of a UAV communication relay in an urban area using the ray tracing method. Kim et al. [9] proposed a path planning strategy of multiple UAV for communication relay between ground control station and a friendly fleet. This work considered communication range and other constraints such as maximum curvature and no fly zone; it was devoted to designing off-line 
trajectories of UAVs with known motion planning of a friendly fleet. Basu et al. [10] investigated the optimal number and placement of UAVs as well as heuristic flocking algorithm, in order to connect all mobile ground nodes. In this work, connectivity between nodes is characterised by only communication range. Zhu et al. [11] proposed an online optimisation algorithm of the location and movement of UAVs to improve the connectivity of a wireless network. They considered a realistic wireless communication model, four different types of network connectivity, and the speed constraints for optimal UAV movement. However, this study investigated the effect of a single UAV only, and the turning constraint of the UAV is not addressed, resulting in discontinuous trajectories.

With this background, this paper proposes high-level deployment algorithms to optimise the trajectory of multiple UAVs for improving the connectivity of a wireless network among a fleet of vessels considering various operational and dynamic constraints of the UAV. In this study, network connectivity is modelled by the context of MANETs (Mobile Ad hoc NETworks) based on global message connectivity. This connectivity is defined by the probability of successful propagation of commands to all the distributed vessels. Typically, a communication system of vessels has enough transmit power to be able to communicate with one another amongst a fleet within a bounded area in maritime environment. However, as the number of vessels in a fleet increases, the communication network complexity increases significantly; thus ensuring network connectivity for sharing information efficiently amongst them becomes a challenging issue. In such a case, it is required to determine which links should be used to distribute information throughout the entire network such that: i) all the nodes are connected; and ii) the overall network connectivity is maximised in terms of global message connectivity.

To achieve aforementioned objectives, the concept of minimum spanning tree (MST) from graph theory [12] is used to obtain the highest probability of a suc- 
cessful transmission using minimum possible links. To further improve network connectivity along with this MST, UAVs equipped with wireless transceivers can be involved as communication relay in a wireless ad hoc network, whilst flying over a fleet of vessels and communicating with other ground nodes as well as other UAVs. Exploiting UAVs is advantageous in that: i) there is less signal attenuation in ground-to-air communication compared to ground-to-ground case suffering from obstacles such as terrain or waves; and ii) UAVs are generally faster than vessels and thus can be rapidly deployed whenever needed, e.g. when there is a partial communication link failure. It is worth noting that UAVs that are able to hover, usually rotorcrafts, might be a good choice to be deployed for stationary communication nodes. In this case, vehicle speed and operational ranges are limited. On the other hand, a fixed-wing UAV can be much faster and operated in much wider areas than those of rotorcrafts; however, it has dynamic and kinematic constraints represented as the minimum (or stall) velocity and turning radius. These constraints should be addressed for the use of fixed-wing UAVs as communication relay.

This paper firstly deals with the UAV deployment for stationary communication nodes, which finds the fixed optimal location of UAVs ensuring the maximum network connectivity. Then, considering movement of vessels and constraints of the fixed-wing UAV in a dynamic environment, this study proposes a nonlinear model predictive control (NMPC) based trajectory planning strategy. By exploiting motion estimates of vessels and states of UAVs, the NMPC algorithm finds a control input sequence for a certain time horizon which optimises network connectivity. Collision between UAVs is also included in the cost function of the optimisation process. In this NMPC frame, since it would be almost infeasible to optimise the control inputs of entire multiple UAVs in a centralised system at once, this study uses a fully-decentralised NMPC concept; each UAV optimises its controller individually based on the future state predictions of the other UAVs. Numerical 
simulations using multiple UAVs as communication relay are performed for both stationary and mobile node case with a fleet of vessels to verify the feasibility and benefits of the proposed approach.

The overall structure of this paper is given as follows. Section 2 explains UAV, vessels and sensor model and the tracking filter used in this study. Section 3 introduces a wireless communication model and modelling of network connectivity using the concept of MST. Section 4 proposes optimisation algorithms for UAV position and trajectory to maximise network connectivity. Section 5 presents numerical simulation results to communication relay scenarios depending on the number of vessels and available UAVs. Lastly, conclusions and future work are given in Section 6.

\section{Problem Definition}

\subsection{UAV dynamic model}

Assuming each UAV has a low-level flight controller such as SAS (Stability Augmentation System) and CAS (Controllability Augmentation System) for heading and velocity hold functions, this study aims to design guidance inputs to this low-level controller for efficient communication relay. Consider a two-dimensional UAV kinematic model [13] as:

$$
\left(\begin{array}{c}
\dot{x} \\
\dot{y} \\
\dot{\psi} \\
\dot{v} \\
\dot{\omega}
\end{array}\right)=f(\mathbf{x}, \mathbf{u})=\left(\begin{array}{c}
v \cos \psi \\
v \sin \psi \\
\omega \\
-\frac{1}{\tau_{v}} v+\frac{1}{\tau_{v}} u_{v} \\
-\frac{1}{\tau_{\omega}} \omega+\frac{1}{\tau_{\omega}} u_{\omega}
\end{array}\right)
$$

where $\mathbf{x}=(x, y, \psi, v, \omega)^{T}$ are the inertial position, heading, speed and yaw rate of the UAV, respectively. $\tau_{v}$ and $\tau_{\omega}$ are time constants for considering actuator 
delay, which can be determined experimentally by analysing the characteristics of the UAV autopilot. $\mathbf{u}=\left(u_{v}, u_{\omega}\right)^{T}$ are the commanded speed and turning rate constrained by the following dynamic limits of fixed-wing UAV:

$$
\begin{aligned}
\left|u_{v}-v_{0}\right| & \leq v_{\max } \\
\left|u_{\omega}\right| & \leq \omega_{\max }
\end{aligned}
$$

where $v_{0}$ is a nominal speed of the UAV. The continuous UAV model in Eq. (1) can be discretised by Euler integration into:

$$
\mathbf{x}_{k+1}=f_{d}\left(\mathbf{x}_{k}, \mathbf{u}_{k}\right)=\mathbf{x}_{k}+T_{s} f\left(\mathbf{x}_{k}, \mathbf{u}_{k}\right)
$$

where $\mathbf{x}_{k}=\left(x_{k}, y_{k}, \psi_{k}, v_{k}, \omega_{k}\right)^{T}, \mathbf{u}_{k}=\left(u_{v k}, u_{\omega k}\right)^{T}$, and $T_{s}$ is a sampling time. Note that the effect of wind is not considered in this study.

\subsection{Marine vessel and sensor model}

This study considers acceleration dynamics to estimate the movement of marine vessels or ground vehicles. This dynamic model defines the target acceleration as a correlated process with a decaying exponential autocorrelation function, which means if there is a certain acceleration rate at a time $t$ then it is likely to be correlated via the exponential at a time instant $t+\tau$. A discretised system equation for the acceleration model is thus expressed in the form:

$$
\mathbf{x}_{k}^{v}=F_{k} \mathbf{x}_{k-1}^{v}+\eta_{k}
$$

here the state vector is $\mathbf{x}_{k}^{v}=\left(x_{k}^{v}, \dot{x}_{k}^{v}, \ddot{x}_{k}^{v}, y_{k}^{v}, \dot{y}_{k}^{v}, \ddot{y}_{k}^{v}\right)^{T}$, and where $\eta_{k}$ is a process noise which represents the acceleration characteristics of the target. The state tran- 
sition matrix $F_{k}$ is given by:

$$
F_{k}=\left[\begin{array}{cccccc}
1 & T_{s} & \Phi & 0 & 0 & 0 \\
0 & 1 & \frac{\left(1-e^{-\alpha_{v} T_{s}}\right)}{\alpha_{v}} & 0 & 0 & 0 \\
0 & 0 & e^{-\alpha T_{s}} & 0 & 0 & 0 \\
0 & 0 & 0 & 1 & T_{s} & \Phi \\
0 & 0 & 0 & 0 & 1 & \frac{\left(1-e^{-\alpha_{v} T_{s}}\right)}{\alpha_{v}} \\
0 & 0 & 0 & 0 & 0 & e^{-\alpha_{v} T_{s}}
\end{array}\right]
$$

where $\Phi=\left(e^{-\alpha_{v} T_{s}}+\alpha_{v} T_{s}-1\right) / \alpha_{v}^{2}$, and $\alpha_{v}$ is a correlation parameter which models different classes of manoeuvring targets. The details of the covariance matrix $Q_{k}$ of the process noise $\eta_{k}$ and other characteristics of this model can be found in [14].

Assuming that global $x$ and $y$ positions of vessels are available to UAVs as measurements using GPS (global positioning system) or other type of sensors, the sensor model can be expressed as:

$$
\mathbf{z}_{k}=H_{k} \mathbf{x}_{k}^{t}+\nu_{k}
$$

where the measurement matrix is $H_{k}=\left[\begin{array}{cccccc}1 & 0 & 0 & 0 & 0 & 0 \\ 0 & 0 & 0 & 1 & 0 & 0\end{array}\right]$. The covariance of measurement noise $\nu_{k} \sim N\left(0, R_{k}\right)$ can be expressed as:

$$
R_{k}=\left[\begin{array}{cc}
\sigma_{x}^{2} & 0 \\
0 & \sigma_{y}^{2}
\end{array}\right]
$$

where $\sigma_{x}$ and $\sigma_{y}$ are the standard deviations of the position of $x$ and $y$, respectively.

\subsection{Tracking filter}

To estimate the states (position, velocity, and acceleration) of vessels, the Kalman filter $(\mathrm{KF})$ is designed. The estimates of states will be used to predict the future 
positions of the vessel for a model-based optimal UAV trajectory planning in Section 4.2. For a general discrete KF, two steps are required: i) prediction step where extrapolation of states and associated error covariance matrix is performed using vessel dynamics as in Eq. (5); and ii) update step where correction is performed which produces a minimum variance estimate of the states with an associated error covariance matrix, using measurements and a sensor model as in Eq. (7). Depending on the type of sensors, different filtering techniques (e.g. nonlinear estimation if sensor measurements are range and/or bearing angle) can be used, and sensor fusion can also be implemented combining data from other sensors or UAVs to improve the estimation accuracy $[13,15]$.

\section{Communication Network Connectivity}

In this study, network connectivity is modelled in the context of MANETs (Mobile Ad hoc NETworks) based on global message connectivity. This connectivity is defined by the probability of successful propagation of commands to all the distributed vessels. This section introduces a realistic wireless communication model and how to achieve efficient network connectivity using the concept of minimum spanning tree.

\subsection{Realistic wireless communication model}

Most previous communication relay research using UAVs have used a simplified wireless communication model considering a communication range only. This work adopts a more realistic wireless communication model between vessel nodes and UAVs, based on the work of [11]. First of all, suppose node $i$ transmits signals

with transmission power $P_{i}$ and observes noise of average power $\sigma_{i}^{2}$ when operating as a receiver. In military applications, it can be assumed that the bandwidth is 
sufficient, and interference is trivial as different users and UAVs use different frequencies. The received SNR (signal-to-noise ratio) $\Gamma_{i j}$ for the signal transmitted by the $i$-th node and received by the $j$-th node is given as:

$$
\Gamma_{i j}=\frac{P_{i} G_{i j}}{\sigma_{j}^{2}}
$$

where the channel gain $G_{i j}$ is expressed as:

$$
G_{i j}=\frac{C_{i j}\left|h_{i j}\right|^{2}}{D_{i j}^{\alpha}}
$$

where $C_{i j}$ is a constant related to antenna gains and shadowing, $h_{i j}$ accounts for multipath fading, $D_{i j}$ is the distance between two nodes, and $\alpha$ is the propagation loss factor. For a successful transmission with an acceptably small packet loss, the SNR needs to be sufficiently high, or in turn, higher than a required minimal level of link quality $\gamma$. Therefore, if the channel has fast Rayleigh fading, i.e. $h_{i j}$ is complex Gaussian with zero mean and unit variance, the probability of a successful transmission between two nodes $i$ and $j$ can be represented as:

$$
P_{r}^{i j}(\Gamma \geq \gamma)=\exp \left(-\frac{\sigma_{j}^{2} \gamma D_{i j}^{\alpha}}{C_{i j} P_{i}}\right)
$$

Note that, if $P_{r}^{i j}$ is smaller than a certain threshold, two nodes might be regarded as disconnected; however, this study assumes that the entire network is connected to each other with a reasonably strong transmission power and focuses on how to improve connectivity exploiting this probability $P_{r}^{i j}$ as the network evolves.

\subsection{Network connectivity using the concept of MST}

As the number of vessels in a fleet increases, the communication network complexity increases significantly; thus ensuring network connectivity for sharing information efficiently amongst them becomes a challenging issue. In such a case, 
the concept of minimum spanning tree (MST) from graph theory can be used to obtain the highest probability of a successful transmission using minimum possible links. The definition of a spanning tree is a subgraph that is itself a tree and connects all the vertices of the graph together. Even if a single graph can have many different spanning trees, a appropriate weight can be assigned to each edge, and the total cost of a given spanning tree can be computed by summing all the weights. An MST is then determined as a spanning tree with the cost that is less than or equal to the cost of every other spanning tree. This study sets the weight of each graph as a function of the aforementioned probability of a successful transmission in Eq. (11) as:

$$
W_{i j}=-\log P_{r}^{i j}
$$

The smaller the weight is, the higher the probability of a successful transmission is, and then, the more likely a message will be delivered to all nodes in the network. To find the MST solution, a variety of polynomial-time algorithms are available, and this study uses the Kruskal algorithm [12].

\section{Optimal UAV Deployment and Trajectory Planning}

To further improve network connectivity along with MST, small UAVs equipped with wireless transceivers can be used as communication relay, while flying over a fleet of vessels and communicating with other ground nodes (vessels) as well as other UAVs via a wireless ad hoc network. This section first presents an optimal UAV deployment (positioning) strategy for stationary communication nodes (i.e. static vessels and hovering UAVs); and then an optimal trajectory planning strategy for fixed-wing UAVs follows for mobile nodes based on a nonlinear model predictive control concept. Figure 1 illustrates an overall flowchart of the proposed approach for communications relay among a fleet of stationary naval vessels. Es- 
pecially, the communications relay for mobile marine vessel nodes requires the motion prediction of them. As shown in Fig. 1, each UAV carries out the estimation/prediction for the state of the marine vessels using GPS and/or other sensor measurements, optimizes its trajectory for global message connectivity, and then decides its control input for optimal positioning. In the mean time, information from the other UAVs is fused with its own estimation in a decentralized way along with the collision avoidance amongst UAVs.

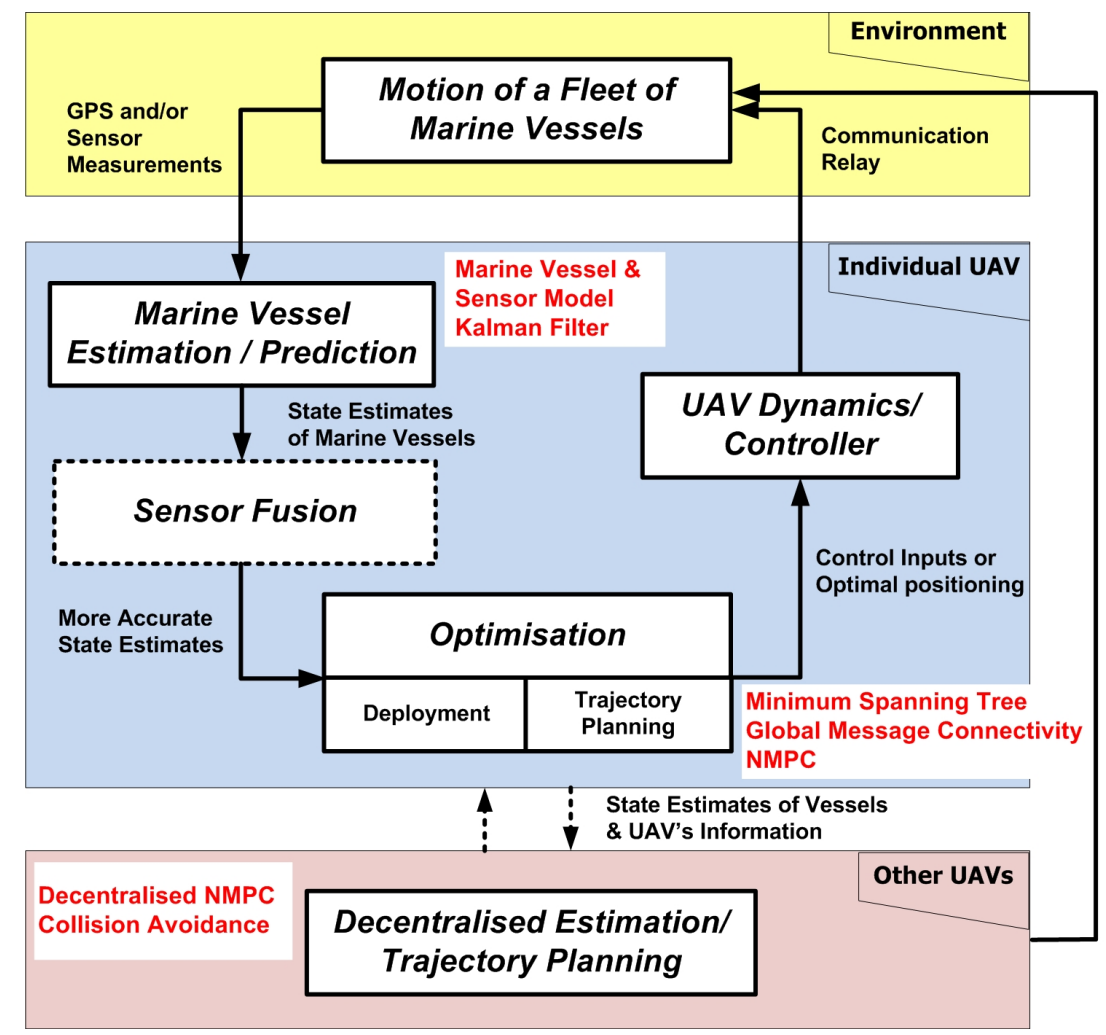

Figure 1: A flowchart of optimal UAV deployment and trajectory planning for communications relay among a fleet of stationary naval vessels 


\subsection{Optimal UAV deployment for stationary nodes}

In order to find the optimal locations of UAVs functioning as communication relay at a fixed position among stationary vessels, let us first define the performance index for network connectivity. This study uses the definition of the global message connectivity which represents the probability that a message can successfully be transmitted to all nodes via the MST. If the positions of UAVs and vessels are given, the MST can be constructed with the aforementioned weight $W_{i j}$ as in Eq. (12) for $n$ UAVs and $m$ vessels. Let us the matrix $A^{\prime} \in R^{(n+m) \times(n+m)}$ represent the adjacency matrix of the MST for a given configuration, then $A_{i j}^{\prime}=1$ if the link

from node $i$ to node $j$ is the part of the MST, and $A_{i j}^{\prime}=0$, otherwise. Since the sum of the weights in the MST corresponds to the overall probability that a message is successfully transmitted via this MST, the performance index for the global message connectivity can be set as:

$$
J_{s}\left(\overline{\mathbf{x}}^{\text {pos }}, \overline{\mathbf{x}}^{v, p o s}\right)=\sum_{i=1}^{n+m} \sum_{j=1}^{n+m} A_{i j}^{\prime} W_{i j} .
$$

Note that the MST $A^{\prime}$ and $W_{i j}$ are determined according to the locations of $n$ UAVs and $m$ vessels, represented as $\overline{\mathbf{x}}^{\text {pos }} \in R^{2 \times n}$ and $\overline{\mathbf{x}}^{v, p o s} \in R^{2 \times m}$, respectively. Then, the UAV deployment problem can be formulated as minimising the performance index for the global network connectivity with respect to the UAV locations $\overline{\mathbf{x}}^{\text {pos }}$ as:

$$
\min _{\overline{\mathbf{x}}^{p o s}} J_{s}\left(\overline{\mathbf{x}}^{\text {pos }}, \overline{\mathbf{x}}^{v, p o s}\right)=\min _{\overline{\mathbf{x}}^{\text {pos }}} \sum_{i=1}^{n+m} \sum_{j=1}^{n+m} A_{i j}^{\prime} W_{i j} .
$$

Note that the implementation of this deployment optimisation process is centralised for a stationary environment, which can be run either in one of UAVs or a mission control station. Once the optimal locations are determined, UAVs are deployed to loiter around those points due to their turning constraints, resulting in a standoff orbit as described in [15]. 


\subsection{Optimal UAV trajectory planning for mobile nodes}

Considering movement of vessels and constraints of the fixed-wing UAV in a dynamic environment, this section proposes a nonlinear model predictive control (NMPC) based trajectory planning strategy. By exploiting motion estimates of vessels obtained from sensor data with filtering techniques (explained in Section 2), as well as states of UAVs, the NMPC algorithm finds a control input sequence $U_{k}^{i}=$ $\left\{\mathbf{u}_{0}^{i}, \mathbf{u}_{1}^{i}, \ldots, \mathbf{u}_{N-1}^{i}\right\} \in R^{2 \times N}$ for the $i$-th UAV, which minimises the following performance index:

$$
\begin{array}{ll}
J_{d}= & \phi\left(\overline{\mathbf{x}}_{N}, \overline{\mathbf{x}}_{N}^{v}\right)+\sum_{k=0}^{N-1} L\left(\overline{\mathbf{x}}_{k}, \overline{\mathbf{x}}_{k}^{v}, \mathbf{u}_{k}^{i}\right) \\
\text { s.t. } & \mathbf{x}_{k+1}^{i}=f_{d}\left(\mathbf{x}_{k}^{i}, \mathbf{u}_{k}^{i}\right) \\
& v_{\min } \leq u_{v k}^{i} \leq v_{\max } \\
& \omega_{\min } \leq u_{\omega k}^{i} \leq \omega_{\max } \\
& \left|C\left(\mathbf{x}_{k}^{i}-\mathbf{x}_{k}^{j \neq i}\right)\right|>r_{c}, \quad \forall j \in\{1, \cdots, n\}
\end{array}
$$

where

$$
\begin{aligned}
\phi\left(\overline{\mathbf{x}}_{N}, \overline{\mathbf{x}}_{N}^{v}\right)= & p_{c} J_{s}\left(\overline{\mathbf{x}}_{N}^{\text {pos }}, \overline{\mathbf{x}}_{N}^{v, \text { pos }}\right) \\
L\left(\overline{\mathbf{x}}_{k}, \overline{\mathbf{x}}_{k}^{v}, \mathbf{u}_{k}^{i}\right)= & \frac{1}{2}\left\{q_{c} J_{s}\left(\overline{\mathbf{x}}_{k}^{\text {pos }}, \overline{\mathbf{x}}_{k}^{v, p o s}\right)\right. \\
& \left.+r_{v}\left(\frac{u_{v k}^{i}-v_{0}}{v_{\max }}\right)^{2}+r_{\omega}\left(\frac{u_{\omega k}^{i}}{\omega_{\max }}\right)^{2}\right\} .
\end{aligned}
$$

Inhere, a superscript $i$ corresponds to the value of the $i$-th $\mathrm{UAV}, J_{s}$ represents the performance index for the global network connectivity as in Eq. (13), $\overline{\mathbf{x}}_{k}^{\text {pos }}=$ $\left\{C \mathbf{x}_{k}^{1}, \cdots, C \mathbf{x}_{k}^{n}\right\}$ and $\overline{\mathbf{x}}_{k}^{v, p o s}=\left\{C_{v} \mathbf{x}_{k}^{v 1}, \cdots, C_{v} \mathbf{x}_{k}^{v m}\right\}$ represent the positions of the current UAVs and the vessels (which will be propagated using the prediction step of the Kalman filter), respectively. $C \in R^{2 \times 5}$ and $C_{v} \in R^{2 \times 6}$ are simply represented by the definition of state variables of UAVs and vessels: $\mathbf{x}_{k}=$ 


$$
\begin{gathered}
\left(x_{k}, y_{k}, \psi_{k}, v_{k}, \omega_{k}\right)^{T} \text { and } \mathbf{x}_{k}^{v}=\left(x_{k}^{v}, \dot{x}_{k}^{v}, \ddot{x}_{k}^{v}, y_{k}^{v}, \dot{y}_{k}^{v}, \ddot{y}_{k}^{v}\right)^{T}: \\
C=\left[\begin{array}{ccccc}
1 & 0 & 0 & 0 & 0 \\
0 & 1 & 0 & 0 & 0
\end{array}\right], C_{v}=\left[\begin{array}{cccccc}
1 & 0 & 0 & 0 & 0 & 0 \\
0 & 0 & 0 & 1 & 0 & 0
\end{array}\right] .
\end{gathered}
$$

Also, $N$ is the length of receding horizon, $v_{o}$ is a nominal speed of UAVs, and $r_{c}$ is a safe distance between the UAVs to prevent collision. $p_{c}, q_{c}, r_{v}$ and $r_{\omega}$ are constant weighting factors. Note that this process is repeated at every sampling time, and thus only the first control input out of a control input sequence $U_{k}^{i}$ is implemented to control the corresponding UAV.

In above NMPC formulation, since it would be almost infeasible to optimise the control inputs of entire multiple UAVs in a centralised system at once, this study uses a fully-decentralised NMPC concept. Each UAV independently determines the MST and optimises its controller using the future state predictions of the other UAVs and the estimates of all vessels, under the assumption that required information can be shared between UAVs via communication within a single sampling time. Depending on the capability of a communications relay equipment on-board and the number of UAVs, this assumption can be relaxed by increasing a sampling time. Decentralized optimisation is performed after receiving the control inputs at the previous sampling and the current state of the other UAVs. At each time step, the MST solutions for the entire network to maintain (enforcing the network connectedness) obtained by UAVs would be the same if UAVs share the same positional information. However, the MST of each UAV might intermittently be different from each other due to communications delay and corruption. One of the possible ways to address this issue would be to communicate the MST structure tagged with each UAV's ID among the mobile nodes and to select an MST having the lowest cost in case of the discrepancy between UAVs. Compared to a centralised one, the proposed structure is beneficial in that each UAV performs its 
own optimisation using as best information as possible with a much reduced computation burden while providing a sub-optimal solution. In addition, the proposed decentralised strategy can be viewed as more robust in terms of the mission success since even in case of the failure of some UAVs in the group, remaining UAVs can continue the communication relay mission.

\section{Numerical Simulations}

This section carries out numerical simulations using the proposed optimal UAV deployment and trajectory planning algorithms for a fleet of eight naval vessels. The parameters used for the simulation are shown in Table 1, and the optimisation solution is computed numerically using Matlab. Eight naval vessels are used as an example case in this paper. Note that as the number of vessels increases, time to find a minimum spanning tree solution in the network increases, which subsequently will increase the whole optimisation process time. In addition, it will require higher communication bandwidth for the UAV to obtain information of all vessels. Therefore, the maximum number of vessels the proposed algorithm can cover needs to be further investigated considering given computation and communication resources.

There are also trade-offs between performance and sampling time. The faster the sampling frequency is, the better performance on the global message connectivity it might have, but the heavier computation load the NMPC requires due to the larger number of receding horizon steps for a given period. Since the surface mobile vessels move with lower speed than the UAVs in the air, the frequency to produce a guidance command for communications relay could be lower than that for control or observation action. In this regard, a sampling time of 0.5 second is used for this simulation; however, a shorter sampling period would be required 
to cover the communications relay among fast-moving ground robots or surface vessels. In a real application, the sampling time for predictive control should be decided by taking into account the number of UAVs and their on-board computation capability.

As shown in Fig. 2(a), the eight vessels are spread out in the area of $1 \mathrm{~km}$ by $1 \mathrm{~km}$. The lines of Fig. 2(a) represent all available communication channels among the vessels, and the numbers on each line display the probability of a successful transmission $W_{i j}$ of each channel. The red lines in Fig. 2(a) represent the final MST of eight vessels without UAVs. The total cost of the global message connectivity is $J=4.3040$ in this case.

Figure 2(b) shows the optimised MST connection of the fleet with a single UAV engaged for communication relay. With this optimised positioning of the UAV, the total cost of the global message connectivity dramatically decreases to $J=2.7437$ compared to $J=4.3040$ with no UAV engagement. This means that deployment of only one UAV node for naval vessels improves $156 \%$ of communication quality. The more UAVs are engaged, the smaller the cost of global message connectivity (equivalent to the higher the probability of successful transmission) is obtained, as shown in Fig. 2(c) and Fig. 3(a). However, note that as the number of UAVs increases, the improvement rate of the connectivity becomes insignificant as can be seen in Fig. 3(a). In this figure, it can also be observed that the cost of global message connectivity varies depending on the transmission power $P_{i}$, and the less power the communication nodes have, the more improvement it can be obtained by the deployment of UAVs as communication relay.

Figure 3(b) shows time histories of the total connectivity cost for a fleet of manoeuvring naval vessels with random noise inputs using the proposed NMPC-based trajectory planning algorithm. Similar to the stationary node case, as the number of UAVs increases, communication quality improves significantly. Figure 4 shows 


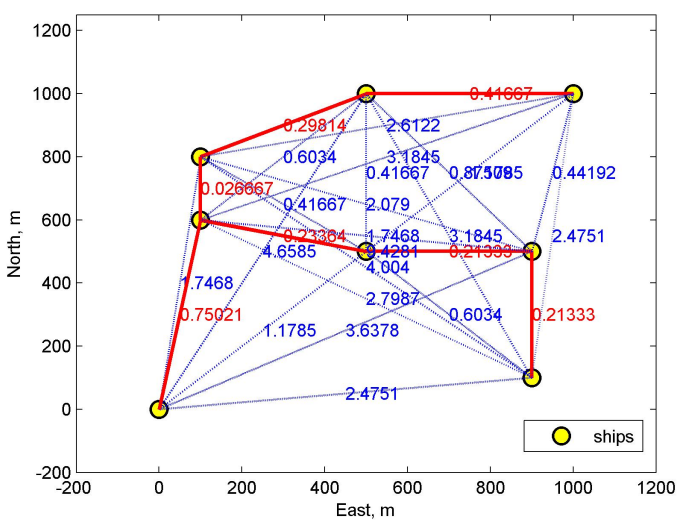

(a) No UAV

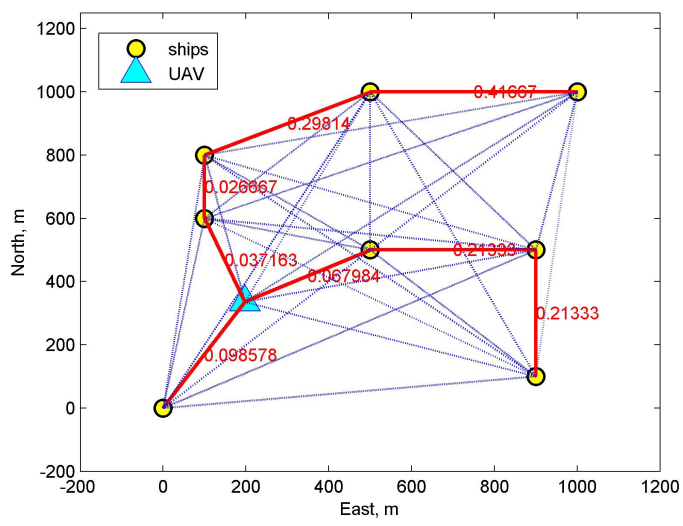

(b) One UAV

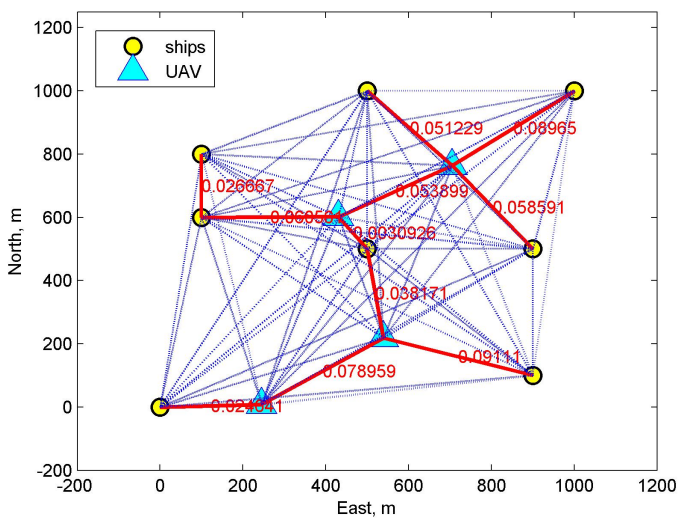

(c) Four UAVs

17

Figure 2: Optimal UAV deployment result and the MST connection for a fleet of stationary naval vessels 


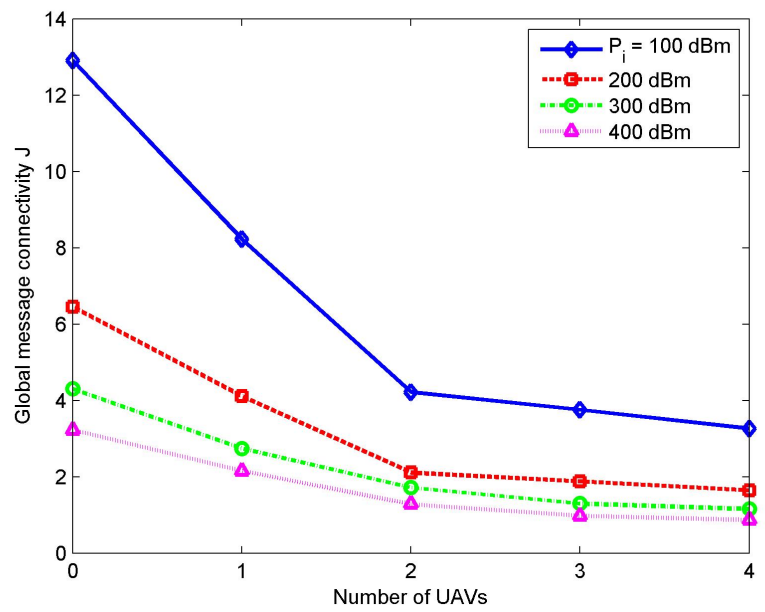

(a) Stationary nodes

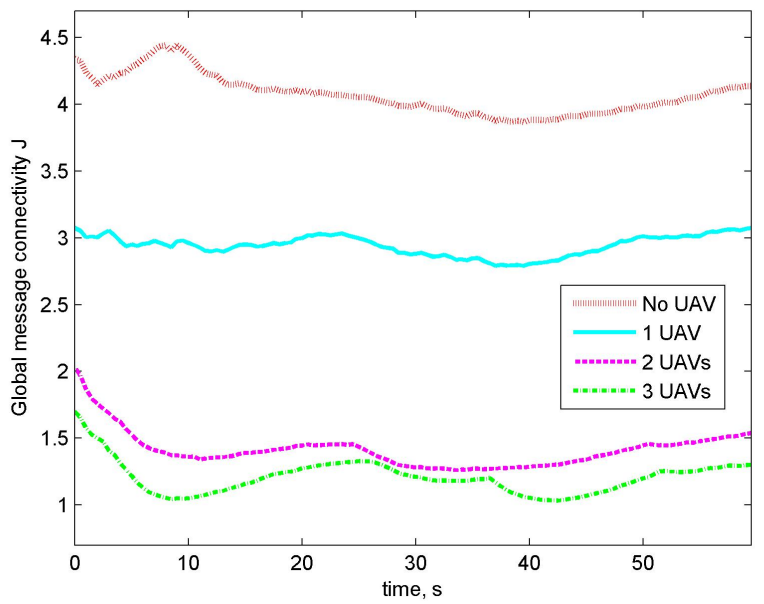

(b) Mobile nodes

Figure 3: The cost of total global message connectivity 
Table 1: Simulation parameters

\begin{tabular}{llc} 
Parameter & Value & Unit \\
\hline Sampling time $T_{s}$ & 0.5 & $\mathrm{sec}$ \\
Receding horizon step $N$ & 5 & $\mathrm{~N} / \mathrm{A}$ \\
Sensor noise std $\left(\sigma_{x}, \sigma_{y}\right)$ & $(5,5)$ & $\mathrm{m}$ \\
Nominal speed of the UAV $v_{o}$ & 20 & $\mathrm{~m} / \mathrm{s}$ \\
Safe distance between UAVs $r_{c}$ & 50 & $\mathrm{~m}$ \\
Speed constraints $\left(v_{\min }, v_{\max }\right)$ & $(10,30)$ & $\mathrm{m} / \mathrm{s}$ \\
Heading rate constraints $\left(\omega_{\min }, \omega_{\max }\right)$ & $(-0.2,0.2)$ & $\mathrm{rad} / \mathrm{s}$ \\
Actuator delay $\left(\tau_{v}, \tau_{\omega}\right)$ & $1 / 3$ & $\mathrm{sec}$ \\
Weighting factor $\left(p_{c}, q_{c}, r_{v}, r_{\omega}\right)$ & $\left(1 \mathrm{e} 1, p_{c} / \mathrm{N}, 1 \mathrm{e} 4,1 \mathrm{e} 5\right)$ & $\mathrm{N} / \mathrm{A}$ \\
Transmission power $P_{i}$ & 300 & $\mathrm{dBm}$ \\
Noise power $\sigma^{2}$ & $1 \mathrm{e}-7$ & $\mathrm{dBm}$ \\
SNR requirement $\gamma$ & 10 & $\mathrm{~dB}$ \\
Propagation loss factor $\alpha$ & 3 & $\mathrm{~N} / \mathrm{A}$ \\
Antenna gain constant $C_{i j}$ & 1 & $\mathrm{~N} / \mathrm{A}$ \\
\hline
\end{tabular}

the optimal trajectory planning result including trajectories of three UAVs and manoeuvring vessels, and the optimised MST connection at each time instant. By controlling the speed and turning rates given by the optimisation process as shown in Fig. 5, UAVs fly over a fleet of vessels ensuring locally minimum cost of the global message connectivity.

Note that the optimisation process to find trajectory planning commands on speed and heading rates can be computationally intractable. If computational capability of the UAV is not enough for the proposed algorithm to be applied in 


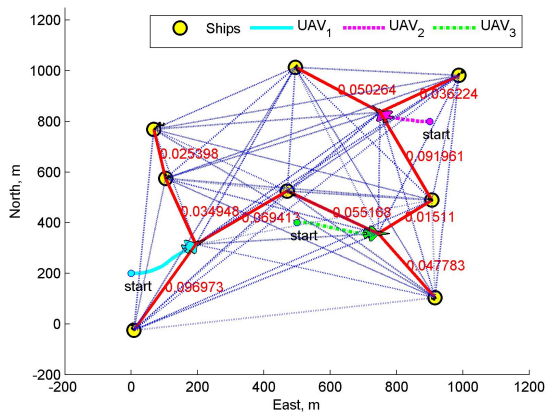

(a) $\mathrm{T}=10 \mathrm{~s}$

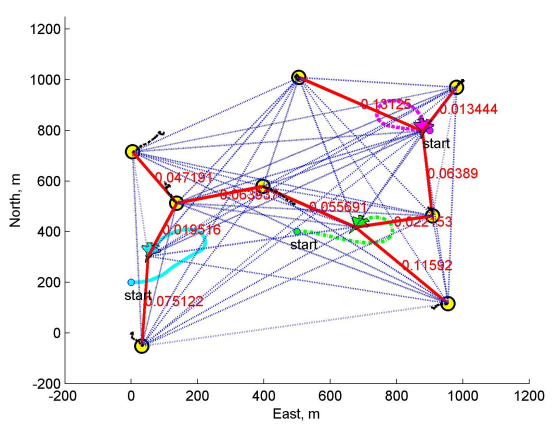

(c) $\mathrm{T}=30 \mathrm{~s}$

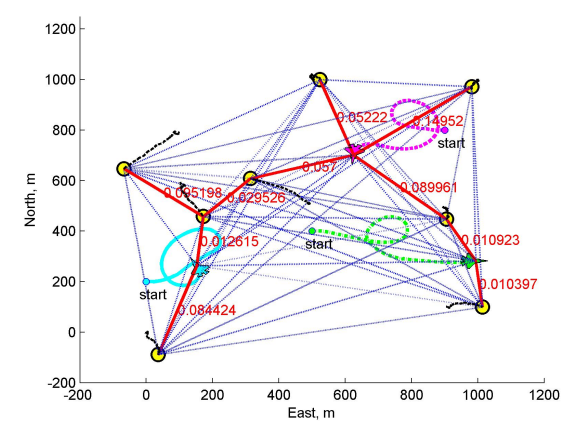

(e) $\mathrm{T}=50 \mathrm{~s}$

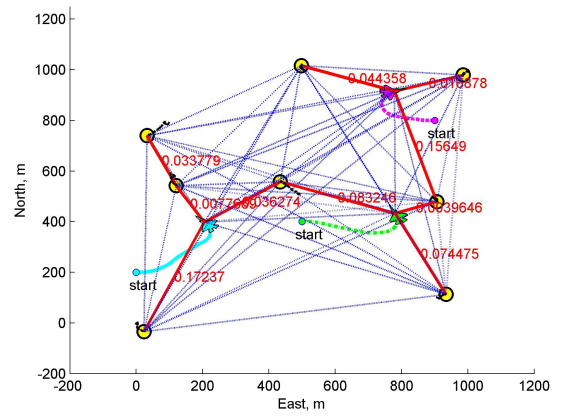

(b) $\mathrm{T}=20 \mathrm{~s}$

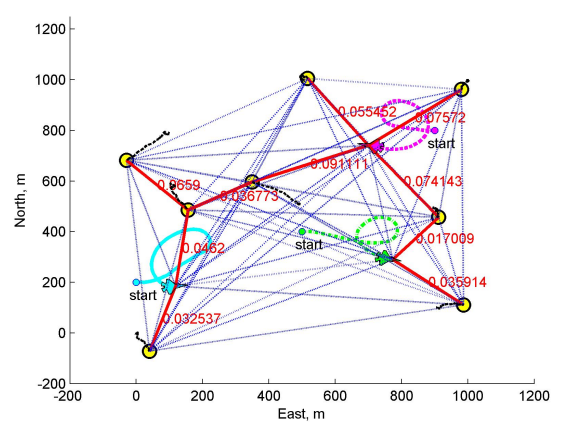

(d) $\mathrm{T}=40 \mathrm{~s}$

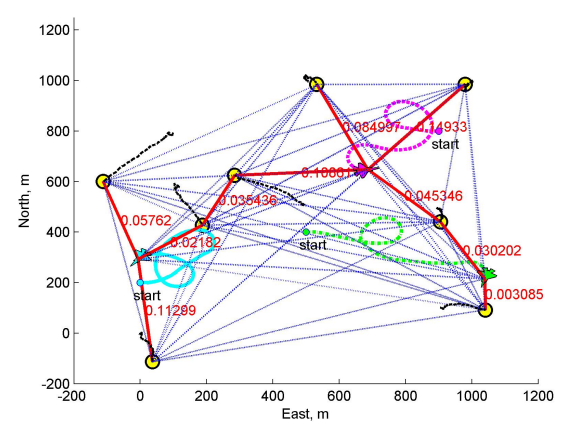

(f) $\mathrm{T}=60 \mathrm{~s}$

Figure 4: Optimal UAV trajectory planning results and the MST connection using three UAVs for a fleet of moving naval vessels 


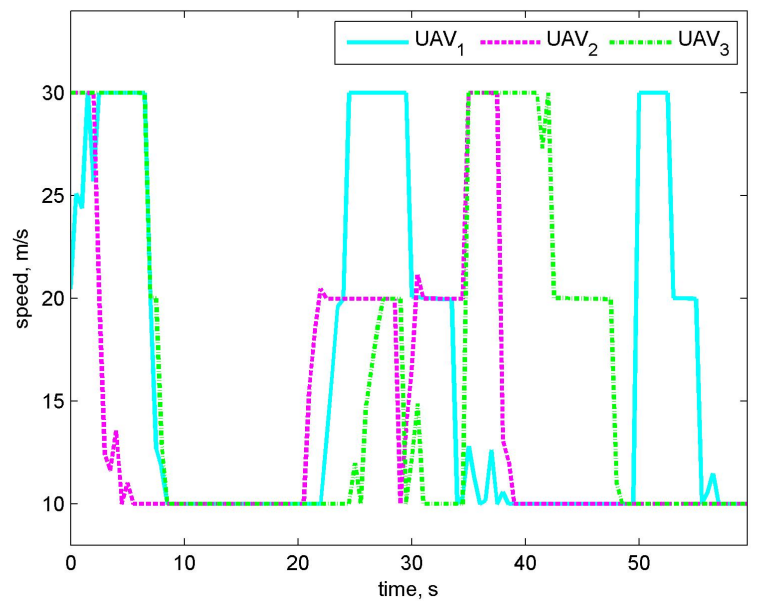

(a) Control input for UAV velocity: $u_{v}$

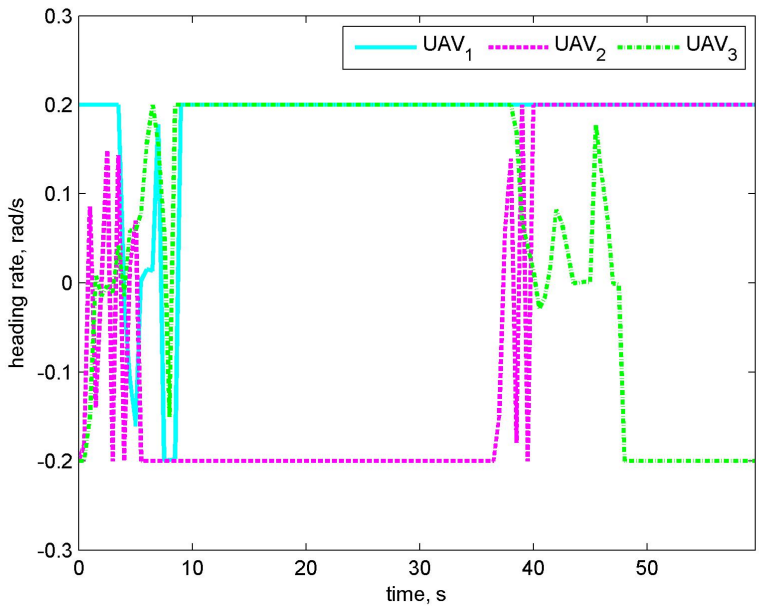

(b) Control input for UAV turn rate: $u_{\omega}$

Figure 5: Control histories of an optimal UAV trajectory planning using three UAVs for a fleet of moving naval vessels 
real-time, several alternatives can be adopted to address this issue by: i) reducing receding horizon step or increasing sampling time step; ii) discretising control inputs into a couple of segmented values. For instance, speed commands can be found among discretised values such as $10,15,20,25,30 \mathrm{~m} / \mathrm{s}$ rather than continuous values between 10 to $30 \mathrm{~m} / \mathrm{s}$. This will reduce computational burden significantly (but degrading the communication quality performance); and iii) if there are too many vessels involved, we could probably split them into several groups (by clustering geographically close vessels into the same group), and then optimise the cost function within the group with a reduced problem dimension. These methods are sacrificing optimality in terms of performance for real-time operation. Above i) and ii) can be applied directly with the proposed framework if a mission scenario and specification are given. However, to apply (iii), further consideration needs to be made, and that will be the future research direction extending the current work.

\section{Conclusions and Future Work}

This paper has presented high-level deployment algorithms to optimise the location and trajectory of multiple UAVs for improving the connectivity of a wireless network amongst a fleet of naval vessels. In particular, a decentralised nonlinear model predictive trajectory planning strategy was proposed to consider movements of vessels and constraints of the fixed-wing UAV in a dynamic environment. Numerical simulation results verified that a mobile ad hoc network in which UAVs are engaged can improve the network connectivity significantly for both static and

dynamic environments. It is assumed that the altitude can be maintained by the autopilot of the UAV. Compared to distance between UAVs and vessels, altitude change resulting from disturbances would be insignificant. Although 3-D trajectory planning with 3-D model can be exploited, it can increase problem dimension 
and computation time which might be detrimental to a NMPC framework to obtain the control inputs in time.

The proposed algorithm allows the location and movement of UAVs to be decided automatically, and thus it increases the quality of network connectivity as well as communication coverage for a mobile ad-hoc network in an efficient manner. Moreover, this work can be easily extended to civilian ground domain for enhancing network connectivity. Research on how to reduce computational complexity of the optimisation process as well as to avoid possible local minimum solutions will be followed as future work of this study. If the frequencies of the guidance law (trajectory planning) and autopilot are not too close, it is common to initially design and verify the guidance law and control algorithm separately, thus this study used simple kinematics for the UAV model. The final validation of the proposed approach with higher complexity simulation models and flight tests remain as another future work.

[1] T. Samad, J. Bay, D. Godbole, Network-centric systems for military operations in urban terrain: The role of UAVs, Proceedings of the IEEE 95 (1) (2007) 92-107.

[2] N. Grumman, www. northropgrumman. com/isr/ (2013).

[3] Thales, www.thalescomminc.com/airborne/ (2013).

[4] M. Hsieh, A. Cowley, V. Kumar, C. Taylor, Maintaining network connectivity and performance in robot teams, Journal of Field Robotics 25 (1) (2008) 111131.

[5] A. Wagner, R. Arkin, Communication-sensitive multi-robot reconnaissance, in: IEEE International Conference on Robotics and Automation (ICRA), New Orleans, LA, 2004, pp. 2480-2487. 
[6] B. Pimentel, M. Campos, Cooperative communication in ad hoc networked mobile robots, in: IEEE/RSJ International Conference on Intelligent Robots and Systems, Las Vegas, NV, 2003, pp. 2876-2881.

[7] M. Pinkney, D. Hampel, S. DiPierro, Unmanned aerial vehicle communication realy, in: IEEE Military Communications Conference (MILCOM'96), McLean, Virginia, 1996.

[8] C. Cerasoli, An analysis of unmanned airborne vehicle relay coverage in urban environments, in: IEEE Military Communications Conference (MILCOM 2007), 2007.

[9] S. Kim, P. Silson, A. Tsourdos, M. Shanmugavel, Dubins path planning of multiple unmanned airborne vehicles for communication relay, Proceedings of the Institution of Mechanical Engineers, Part G: Journal of Aerospace Engineering 225 (1) (2011) 12-25.

[10] P. Basu, J. Redi, V. Shurbanov, Coordinated flocking of UAVs for improved connectivity of mobile ground nodes, in: IEEE Military Communications Conference (MILCOM 2004), 2004.

[11] H. Zhu, A. Swindlehurst, K. Liu, Optimization of MANET connectivity via smart deployment/movement of unmanned air vehicles, IEEE Transactions on Vehicular Technology 58 (7) (2009) 3533-3546.

[12] R. Diestel, Graph theory in Graduate Texts in Mathematics, 3rd Ed., Vol. 173, New York: Springer-Verlag, 2005.

[13] S. Kim, H. Oh, A. Tsourdos, Nonlinear model predictive coordinated standoff tracking of moving ground vehicle, Journal of Guidance, Control and Dynamics 36 (2) (2013) 557-566. 
[14] K. Mehrotra, P. R. Mahapatra, A jerk model for tracking highly maneuvering targets, IEEE Transactions on Aerospace and Electronic Systems 33 (4) (1997) 1094-1105.

[15] H. Oh, S. Kim, A. Tsourdos, B. White, Decentralised road-map assisted ground target tracking using a team of UAVs, in: 9th IET Data Fusion \& Target Tracking, London, UK, 2012. 